has also been suggested ${ }^{14}$. Given that the tumor suppressors p53 and p19 are upregulated in $\mathrm{Bublb} b^{\mathrm{H} / \mathrm{H}}$ senescent cells, it would be interesting to determine whether loss of these in $B u b 1 b^{\mathrm{H} / \mathrm{H}}$ mice would relax cell cycle control, promote tumorigenesis and perhaps alleviate some of the phenotypes associated with aging.

Whether aneuploidy is a specific driving force in the development of cancer is controversial. With the demonstration that severe disruption of $B u b 1 b$ leads to aging-related phenotypes, the study by Baker et al. ${ }^{3}$ now adds aneuploidy and aging to the debate about the relationship between biological cause and consequence. Regardless of these hierarchical considerations, it is apparent that although silencing of the mitotic checkpoint (by pharmacological agents, for example) may be beneficial for inhibiting the growth of cancer cells, the price we may pay is premature aging.

1. Hasty, P., Campisi, J., Hoeijmakers, J., van Steeg, H. \& Vijg, J. Science 299, 1355-1359 (2003).

2. McMurray, M.A. \& Gottschling, D.E. Science 301, 1908-1911 (2003)

3. Baker, D.J. et al. Nat. Genet. 36, 744-749 (2004).
4. Biggins, S. \& Murray, A.W. Curr. Opin. Genet. Dev. $\mathbf{9}$, 230-236 (1999).

5. Wassmann, K. \& Benezra, R. Curr. Opin. Genet. Dev. 11, 83-90 (2001)

6. Hoyt, M.A. J. Cell Biol. 154, 909-911 (2001).

7. Dobles, M., Liberal, V., Scott, M.L., Benezra, R. \& Sorger, P.K. Cell 101, 635-645 (2000).

8. Wang, Q. et al. Blood 103, 1278-1285 (2004).

9. Michel, L. et al. Proc. Natl. Acad. Sci. USA 101 4459-4464 (2004)

10. Kops, G.J., Foltz, D.R. \& Cleveland, D.W. Proc. Natl. Acad. Sci. USA 101, 8699-8704 (2004).

11. Michel, L.S. et al. Nature 409, 355-359 (2001).

12. Dai, W. et al. Cancer Res. 64, 440-445 (2004).

13. Bharadwaj, R. \& Yu, H. Oncogene 23, 2016-2027 (2004).

14. Margolis, R.L., Lohez, O.D. \& Andreassen, P.R. J. Cell. Biochem. 88, 673-683 (2003).

\title{
Resolving schizophrenia's CATCH22
}

\author{
Assen Jablensky \\ Schizophrenia is a severely disabling disorder with a lifetime risk of $\sim 1 \%$, which accounts for $2.8 \%$ of the global \\ burden of disease. A new study provides evidence that a third gene in the $22 q 11$ region is associated with \\ schizophrenia, further confounding an already complicated story.
}

A genetic contribution to the etiology of schizophrenia is well established, and environmental exposures, from neurodevelopmental insults to psychosocial factors, are also suspected to have a role. The search for associated genes and pathogenetic pathways is hampered by complex genetics, lack of a pathognomonic neuropathology or neurophysiology, and a phenotype defined solely by psychopathological criteria (e.g., delusions and hallucinations). More than 30 genome scans and numerous association studies have resulted in multiple suggestive linkage findings and tentative associations ${ }^{1,2}$. Further insights are being gained through studies bridging linkage and association studies with gene expression and functional effects in experimental animals that model aspects of the disease phenotype. On page 725 of this issue, Jun Mukai and colleagues ${ }^{3}$ report on a new candidate gene, $Z D H H C 8$, in the $\sim 3-\mathrm{Mb}$ region on chromosome 22q.11.2, which is heterozygously deleted in DiGeorge/velocardiofacial syndrome, also known by the particularly apt acronym CATCH22 (cardiac abnormality/abnormal facies, T-cell deficit, cleft palate, hypocalcemia; OMIM 188400).

Assen Jablensky is at the School of Psychiatry and Clinical Neurosciences, University of Western Australia, Perth, Australia.

e-mail:assen@cyllene.uwa.edu.au

\section{From association to knockout}

The 22q.11 microdeletion region is associated with increased risk for a range of neuropsychiatric phenotypes, including schizophrenia, rapidly cycling affective bipolar disorder, learning disability, attention

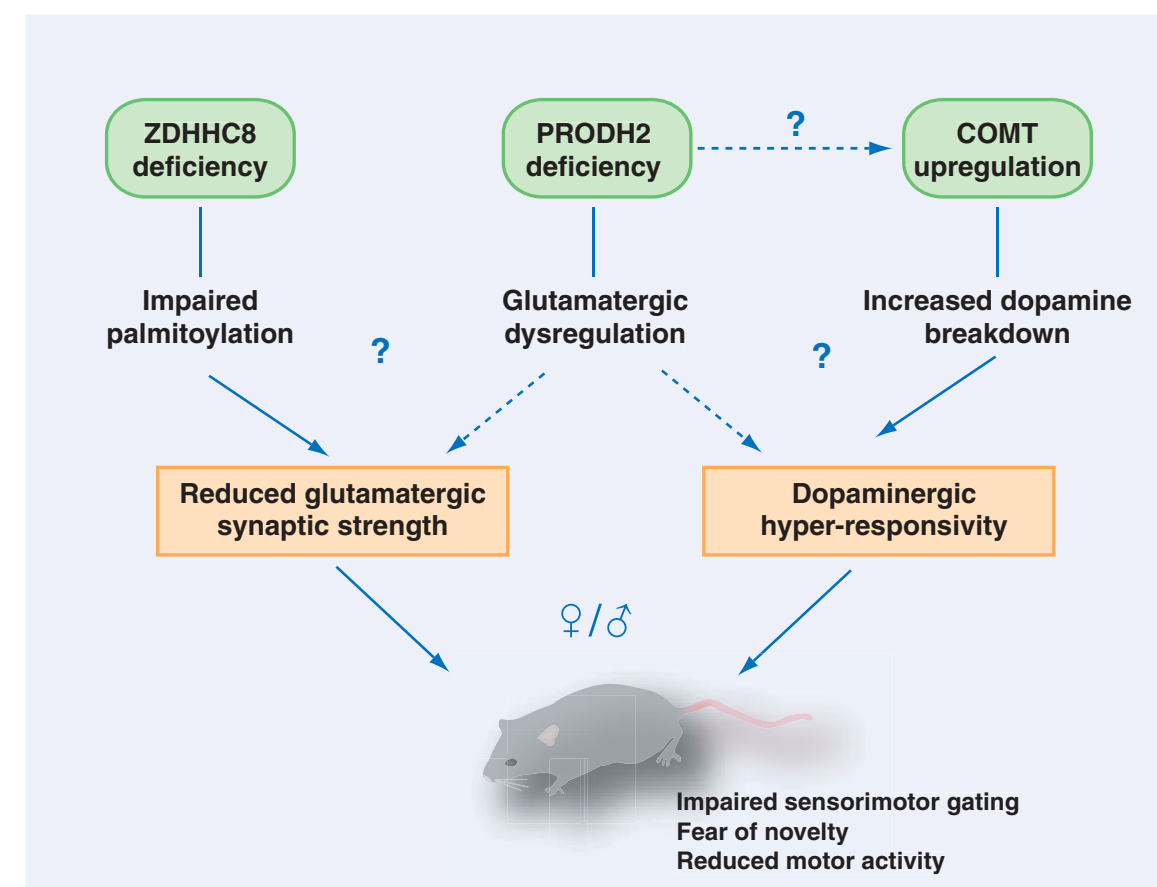

Figure 1 A deficiency in any of three schizophrenia-associated genes on 22q11 can result in behavioral impairments in knockout or heterozygous mice. Further dissection of independent and synergistic effects of these genes in animal models, and endophenotype refinement in individuals with schizophrenia and in controls, will be required to fully delineate the causative pathways. 
deficit disorder, anxiety and characterological peculiarities such as shyness or impulsiveness. It contains at least three genes (COMT, PRODH2 and ZDHHC8) implicated in schizophrenia risk. Studies of rare but well characterized syndromes, such as 22q11-related schizophrenia, can facilitate formulation of coherent pathogenetic hypotheses, eventually leading to identification of specific causal pathways and advances in the general understanding of complex disorders ${ }^{4}$.

Previously, this group provided evidence that variants at the PRODH2 locus in the microdeletion region on 22q11 contribute to schizophrenia risk ${ }^{5}$. Now, they describe a significant association between schizophrenia and a single-nucleotide polymorphism (SNP) in a new gene in the same region, $Z \mathrm{DHHC}$, encoding a putative new member of the palmitoyltransferase family. This intronic SNP modifies $Z D H H C 8$ expression by causing imperfect splicing, intron retention and reduced enzyme activity. The association between this SNP and schizophrenia was confirmed in three independent family and case-control samples from the United States and South Africa.

$Z d h h c 8$ knockout mice had no morphological brain abnormalities but were deficient in the prepulse inhibition (PPI) measure of the startle reflex paradigm. Both knockout and heterozygous mice were abnormal with respect to indices of fearfulness and rearing behavior and proved resistant to the locomotor-activating effect of the psychomimetic NMDA blocker dizocilpine.

Although the biology of ZDHHC8 is incompletely understood, palmitoylation is known to modulate activity-dependent plasticity at glutamatergic synapses in the cortex and hippocampus ${ }^{6}$. The authors suggest that the behavioral impairments in the mouse may involve interactions among $\mathrm{ZDHHC8}$, PRODH2 and COMT (Fig. 1).

Notably, the abnormal behaviors in Zdhhc8deficient mice were restricted to females. In the family sample, the authors found a preferential transmission of the risk allele in females, indicating that the sex-specific effect is unrelated to differences in the splicing of ZDHHC8. This female predilection is puzzling, because another study ${ }^{7}$ found that $\mathrm{a} \sim 1-\mathrm{Mb}$ deletion in 22q11 (encompassing COMT and PRODH2) produced PPI and fear-conditioning abnormalities in male mice only. Considering that a difference has also been found in the transmission of a schizophrenia-associated polymorphism in $\mathrm{COMT}^{8}$, there is a riddle on $22 \mathrm{q} 11$ waiting for a solution.

\section{Schizophrenic mice?}

It is difficult to envisage how schizophrenia symptoms, such as false beliefs, hallucinations, passivity experiences and thought disorder, could be elicited in nonhuman species. But complex experiential phenomena are probably underpinned by cognitive deficits that may, in part, be reproducible across species. One such phenotype, the startle reflex to sudden intrusive stimuli, involves a well-defined neural circuit engaging nuclei in the brain stem, striatum, pallidum and thalamus, with projections to the cortex. The startle response is attenuated when the triggering stimulus is preceded by a weak prestimulus (prepulse). The degree of PPI is a measure of sensorimotor gating, an automatic filter protecting the brain from flooding with irrelevant stimuli. PPI breakdown is frequent in schizophrenia, but it also occurs in stress-related disorders, alcoholism, neurodegenerative disease and autism ${ }^{9}$. Although PPI deficit is not a specific marker of schizophrenia, it correlates with other measures of attention control and, possibly, thought disorder in affected individuals and is present in a proportion of their unaffected relatives ${ }^{10}$. Together with the capacity to dissect genetic and environmental influences in animals, this is a valid reason to regard PPI as a modular phenotype in schizophrenia research.

\section{Modular endophenotypes}

Schizophrenia geneticists face a catch-22 situation: they seek to identify specific genes contributing to an overinclusive diagnostic category for which no specific biological substrate is identifiable, probably because of admixture between different underlying disease subtypes. It is increasingly likely that current diagnostic classifications, based on symptoms, do not demarcate genetic entities. As the clinical manifestations of schizophrenia might be a common final pathway for several separate variants ${ }^{11}$ or endophenotypes ${ }^{12}$, the quest for specificity of genetic and neurobiological findings in relation to diagnostic categories may be misplaced. Can schizophrenia result from the interaction between several modular endophenotypes, each a distinct pattern of neurocognitive or neurophysiological deficits? Identifying such endophenotypes and their genetic basis is a daunting, but not impossible, task. Because the 22q11 region is associated not only with schizophrenia risk, but also with a variety of other phenotypes, its further dissection by convergent genomic and pathophysiological analysis will provide a better understanding of the genetic architecture of psychiatric disorders on the whole.

1. Sklar, P. Annu. Rev. Genomics Hum. Genet. 3 371-413 (2002)

2. Harrison, P.J. \& Owen, M.J. Lancet 361, 417-419 (2003).

3. Mukai, J. et al. Nat. Genet. 36, 725-731 (2004).

4. Chakravarti, A. Proc. Natl. Acad. Sci. USA 99, 4755-4756 (2002).

5. Liu, H. et al. Proc. Natl. Acad. Sci. USA 99, 3717-3722 (2002).

6. El-Husseini, A.E. et al. Cell 108, 849-863 (2002).

7. Paylor, R. et al. Hum. Mol. Genet. 10, 2645-2650 (2001).

8. Shifman, S. et al. Am. J. Hum. Genet. 71, 1296-1302 (2002).

9. Anonymous. Nat. Neurosci. 6, 323 (2003).

10. Cadenhead, K,S. et al. Am. J. Psychiatry 157, 1660-1668

11. Cromwell R.L. et al. Acta Psychiatr. Scand. 90 Suppl. 384, 34-39 (1994)

12. Gottesman, I.I. \& Gould, T.D. Am. J. Psychiatry 160 , 636-645 (2003). 